\title{
Funciones ejecutivas en estudiantes universitarios con patrón de consumo excesivo de alcohol
}

\section{Executive functions in university students with binge drinking alcohol intake}

\author{
Alejandra Mondragón-Mayal, Yvonne Flores-Medina ${ }^{2}$, \\ Gabriela López-Arreaga ${ }^{l}$, Selzin López-Ramírez ${ }^{I}$ \\ y Francisco Paz-Rodríguez ${ }^{3}$ \\ Universidad Nacional Autónoma de México ${ }^{1}$ \\ Instituto Nacional de Psiquiatría "Ramón de la Fuente Muñiz"2 \\ Instituto Nacional de Neurología y Neurocirugía ${ }^{3}$
}

Autor para correspondencia: Francisco Paz-Rodríguez,fpaz@innn.edu.mx.

\section{RESUMEN}

\begin{abstract}
El patrón de consumo excesivo de alcohol (CEA en lo sucesivo) se define como la ingesta excesiva de alcohol en una misma ocasión, con una frecuencia mínima de una vez al mes. Los jóvenes con CEA son vulnerables a presentar déficits cognitivos; específicamente, alteraciones en funciones ejecutivas, las cuales están estrechamente relacionadas con la maduración y el funcionamiento de los lóbulos frontales. El objetivo de este trabajo fue evaluar el funcionamiento ejecutivo de jóvenes universitarios con CEA mediante una batería especializada para la evaluación de tales funciones. Se evaluó a 39 estudiantes divididos en dos grupos según su ingesta de alcohol: grupo de consumo excesivo de alcohol (GCE), con 20 participantes, y grupo control (GC), con 19. Se utilizó la Batería Neuropsicológica de las Funciones Ejecutivas y los Lóbulos Frontales (BANFE en lo que sigue). Se observaron diferencias significativas en el dominio dorsolateral y en la puntuación total de la BANFE. El GC tuvo un mejor desempeño que el GCE. En el análisis específico de las subpruebas, se observó un desempeño significativamente menor del GCE respecto al GC en tareas de memoria de trabajo verbal y flexibilidad cognitiva, por lo que se concluye que el patrón de consumo excesivo de alcohol tiene un efecto negativo sobre las funciones ejecutivas, especialmente en las asociadas con el área prefrontal dorsolateral, lo que resulta compatible con la hipótesis de la vulnerabilidad del lóbulo frontal al consumo de alcohol.
\end{abstract}

Palabras clave: Consumo excesivo de alcohol; Funciones ejecutivas; Alcohol; Consumo de riesgo; Consumo de sustancias.

\footnotetext{
${ }^{1}$ Carrera de Psicología, Facultad de Estudios Superiores Iztacala, Av. de los Barrios 1, Los Reyes Ixtacala, Barrio de los Árboles/Barrio de los Héroes, 54090 Tlalnepantla de Baz, Edo. de México, México, correos electrónicos: trook79@gmail.com, gabrielalopezarreaga@gmail.com, melistar_tink@hotmail.com.

${ }^{2}$ Servicio de Rehabilitación, Calzada México-Xochimilco Núm. 101, Col. San Lorenzo Huipulco, Tlalpan, 14370 Ciudad de México, México, correo electrónico: yg.floresmedina@gmail.com.

${ }_{3}^{3}$ Departamento de Neuropsicología y Grupos de Apoyo, Insurgentes Sur 3877, Col. La Fama, 14269 Ciudad de México, México, tel. (55)55-28-78-78, correo electrónico: fpaz@innn.edu.mx.
} 


\section{ABSTRACT}

Binge drinking $(B D)$ intake pattern is defined as excessive alcohol consumption in a single occasion, at least once a month. Young adults with BD intake pattern may be at increased risk for cognitive impairment. Particularly, deficits in executive functions strongly relate to frontal lobe maturation and functioning. The objective of the present study was to assess executive functioning of university students with BD intake pattern, using a specialized neuropsychological battery for such functions. Thirty-nine students were included and divided into two groups according to their alcohol intake pattern: $B D$ group $(B D G=20)$ and control group $(C G=19)$. The Neuropsychological Battery of Executive Functions and the Frontal Lobes (NBEFFL) was applied. Significant differences were observed in the dorsolateral domain and NBEFFL's total SCOre, where the CG outperformed the BDG. Specific subtest analyses showed significantly poorer performance of $B D G$ than $C G$ in verbal working memory and cognitive flexibility tasks. In conclusion, BD pattern intake has a negative effect on executive functions, particularly those associated to the dorsolateral prefrontal area. These results are consistent with the frontal lobe vulnerability hypothesis related to alcohol consumption.

Keywords: Binge drinking; Executive functions; Alcohol; Risk intake; Substance abuse.

Recibido: 02/03/2020

Aceptado: 19/06/2020

\section{INTRODUCCIÓN}

$\mathrm{E}$ 1 patrón de consumo excesivo de alcohol (CEA en lo sucesivo) se define como la ingesta excesiva de alcohol (más de cinco copas para hombres o cuatro para mujeres) en una misma ocasión, con una frecuencia mínima de una vez al mes. Tales episodios se mezclan con periodos de abstinencia (Chung, Creswell, Bachrach, Clark y Martin, 2018; Wechsler, Davenport, Dowdell y Rimm, 1995; Wechsler et al., 2002). Este patrón de consumo es frecuente en países latinoamericanos como México, Brasil y Perú (Monteiro, 2007); cabe señalar que en el caso de México, la prevalencia del CEA ha aumentado en los últimos años de $12.3 \%$ en 2011 a $19.8 \%$ en 2016 (Reséndiz et al., 2018). Recientemente, Kulis, Marsiglia, Nuño, Lozano y Medina-Mora (2018) reportaron una prevalencia de CEA en estudiantes de escuelas públicas de $14 \%$.

Diversos estudios epidemiológicos han señalado que el CEA es frecuente en adolescentes y adultos jóvenes en todo el mundo. Datos de países europeos y americanos estiman que la presencia de CEA en jóvenes de 15 a 16 años se mantiene en un rango de 8 a 11\% (Kuntsche, Kuntsche, Thrul y Gmel, 2017). En México, el estudio de Reséndiz et al. (2018) mostró que el CEA aumentó al doble de 2011 a 2016 (de 4.3 a 8.3\%) en jóvenes de entre 12 y 17 años. Asimismo, que en personas mayores de 18 años también aumentó significativamente durante este mismo periodo: de $13.9 \%$ en 2011 a 22.1\% en 2016. De acuerdo con la Encuesta Nacional de Nutrición y Salud (ENSANUT) 2018 (Secretaría de Salud, Instituto Nacional de Salud Pública e Instituto Nacional de Estadística y Geografía, 2018), la población de 10 a 19 años reportó una prevalencia de consumo de alcohol alguna vez en la vida de $21.7 \%$ (mujeres $13.8 \%$, hombres 42.8\%). Las cinco entidades con porcentajes de consumo más elevados fueron Puebla (30.8\%), Colima (30.3\%), Ciudad de México (29.5\%), San Luis Potosí (29.3\%) y Jalisco (29.1\%). De estos consumidores, $11.5 \%$ ingieren bebidas alcohólicas casi todos los días.

Las consecuencias negativas relacionadas con el patrón CEA en adolescentes y adultos jóvenes se han descrito ampliamente. Una revisión de Kuntsche et al. (2017) que estudió el impacto del CEA sobre la salud mostró que este patrón de consumo se relaciona con actividades de riesgo, como mantener relaciones sexuales sin protección, manejar bajo los efectos del alcohol o actuar de forma violenta hacia los demás y hacia sí mismo.

La adolescencia y la adultez temprana son periodos críticos en el neurodesarrollo de los seres humanos. Los hallazgos sobre los procesos de maduración cerebral coinciden en señalar que tanto la poda neuronal, que permite la eliminación de sinapsis poco funcionales, como la mielinización de áreas específicas como la corteza prefrontal, se mantienen hasta la adultez joven y culminan cerca de los 30 años (Lebel y Beaulieu, 2011; López et al., 2014). Las alteraciones en las trayectorias de maduración generadas por agentes tóxicos como el alcohol en esta etapa pueden producir cambios 
estructurales y funcionales, con consecuencias en la conducta de los individuos. Los trabajos de Hermens et al. (2013), Jacobus y Tapert (2013), Squeglia, Jacobus y Tapert (2014), Cservenka y Brumback (2017) y Lees et al. (2020) reportan coincidentemente que en los adolescentes con consumo nocivo de alcohol puede apreciarse una disminución acelerada del volumen de la sustancia gris en estructuras como el hipocampo, la corteza prefrontal y el cerebelo, así como un adelgazamiento de la sustancia blanca que conecta estas estructuras. Tales resultados pueden generar por sí mismos modificaciones en la eficiencia del procesamiento de la información, esto es, tener consecuencias cognitivas directas. Adicionalmente, algunos autores han encontrado que el consumo de alcohol en la adolescencia se asocia con un aumento en la activación de zonas como la corteza insular, la subcallosa y la región anterior del giro del cíngulo cuando existen pistas relacionadas con el consumo. Dicha hiperactividad de las zonas límbicas, asociada a las pistas sobre el consumo de alcohol y la disminución del volumen y la conectividad cortical en zonas de integración de la información y control de la conducta, aumentan en adolescentes con CEA el riesgo de desarrollar un trastorno de dependencia al alcohol (Haller, Handley, Chassin y Bountress, 2010; Lannoy, Billieux, Dormal y Maurage, 2019).

Hay una bibliografía creciente sobre los efectos específicos del CEA y el funcionamiento cognitivo en adolescentes y adultos jóvenes. Se reportan, por ejemplo, fallas específicas en el proceso de atención sostenida o concentración y en la memoria episódica (Hartley, Elsabagh y File, 2004; Mota et al., 2013; Townshed y Duka, 2005), y también alteraciones en las funciones ejecutivas, que son definidas por Stuss y Alexander (2000) como procesos específicos relacionados con diferentes regiones de los lóbulos frontales, que convergen en un concepto general de funciones de control o sistema de supervisión. Tales funciones están relacionadas con la organización de la conducta compleja, la respuesta afectiva, la respuesta social y la metacognición. De acuerdo con Diamond (2013), dichas funciones pueden dividirse en respuesta inhibitoria, control de la interferencia, memoria de trabajo, flexibilidad cognitiva y pla- neación. Se ha reportado que jóvenes con CEA presentan un funcionamiento ejecutivo deficiente en dominios como flexibilidad cognitiva, planificación, automonitoreo, memoria de trabajo, toma de decisiones e inhibición, en comparación con su grupo normativo, lo que concuerda con la sensibilidad del lóbulo frontal al consumo de alcohol (Hartley et al., 2004; Houston et al., 2014; Lannoy, Billieux, Dormal y Maurage, 2019; Lannoy, Maurage, D'Hont, Billieux y Dormal, 2019; Lees et al., 2019; Mota et al., 2013; Parada et al., 2012; Salas et al., 2016; Scaife y Duka, 2009; Townshed y Duka, 2005).

Pese a la gran cantidad de literatura que hay acerca de los déficits cognitivos asociados con el CEA, también existen reportes que resultan inconsistentes con esos hallazgos. Hartley et al. (2004), por ejemplo, exploraron tareas de memoria de trabajo visoespacial entre individuos con y sin CEA y no encontraron diferencias significativas. Boelema et al. (2015) no hallaron diferencias en la maduración del funcionamiento ejecutivo (memoria de trabajo, inhibición y atención) en un estudio longitudinal de niños con distintos patrones de consumo de alcohol (no consumo, consumo leve, consumo excesivo infrecuente, consumo excesivo ascendente, consumo excesivo descendente y consumo excesivo crónico), mismos que fueron evaluados neuropsicológicamente en dos momentos: a los 11 y a los 19 años. En un estudio reciente de Hendricks, van de Rest, Snippe, Kieboom y Hogenelst (2020), en el que se evaluó memoria, planeación y razonamiento en estudiantes universitarios en una primera ocasión con un seguimiento efectuado al año, no se encontró asociación entre el desempeño cognitivo y el consumo de alcohol. En la misma línea, en el estudio de Mahedy et al. (2020) se evaluó memoria de trabajo, inhibición y reconocimiento de emociones en un grupo de participantes con diferentes grados de consumo de alcohol, sin encontrar una asociación entre el desempeño cognitivo y el CEA.

Cabe señalar que, también se ha explorado si hay diferencias en las alteraciones cognitivas relacionadas con el sexo en personas con CEA. Los hallazgos son muy variables, puesto que algunos autores han encontrado efectos negativos del CEA más evidentes en las mujeres: en pruebas de me- 
moria de trabajo espacial (Scaife y Duka, 2009; Townshed y Duka, 2005) y velocidad de procesamiento (Salas et al., 2016). Hartley et al. (2004) observaron que los hombres con CEA mostraban una respuesta más lenta ante una tarea de reconocimiento espacial, en comparación con varones sin CEA, efecto no observado en las mujeres. Boelema et al. (2015) no encontraron que el sexo fuera una variable moderadora del desempeño cognitivo. Algo particularmente interesante es que existen estudios hechos con técnicas de neuroimagen en los que se han apreciado cambios diferenciales del sistema nervioso entre hombres y mujeres con CEA. Squeglia, Schweinsburg, Pulido y Tapert (2011), por medio de resonancia magnética funcional, hallaron una interacción significativa entre el sexo en individuos con CEA en las regiones frontales, temporales y el cerebelo. De hecho, las mujeres con CEA mostraban una menor activación de estas zonas, la cual se relacionó con un bajo desempeño en la atención sostenida y la memoria de trabajo; mientras que hombres con CEA mostraron una mayor activación en tales zonas, asociada a un mejor desempeño en tareas visoespaciales. Squeglia et al. (2012), en un estudio hecho con resonancia magnética, encontraron que las mujeres con CEA tenían un mayor grosor cortical en las zonas frontales izquierdas, lo cual se asocia con problemas visoespaciales, inhibitorios $\mathrm{y}$ atencionales. Los hombres con CEA mostraron menor grosor cortical, que está asociado a un bajo desempeño en tareas de atención. En la revisión sistemática realizada por Carbia, López, Corral y Cadaveira (2018) se señala que la evidencia al respecto, dada la heterogeneidad de los resultados reportados, es poco concluyente.

Considerando las dificultades que hay para comparar los estudios, es probable que utilizar instrumentos específicos para la evaluación de las funciones ejecutivas sea de especial utilidad para caracterizar el perfil cognitivo de los jóvenes con CEA. Por esta razón, el objetivo de este trabajo fue evaluar el funcionamiento ejecutivo en jóvenes universitarios con patrón de CEA, y compararlo con un grupo de jóvenes sin ese patrón, utilizando para ello una batería neuropsicológica especializada para evaluar tales funciones.

\section{MÉTODO}

\section{Participantes}

Se reclutó originalmente a 105 participantes (39 mujeres y 67 hombres), con un rango de edad de 18 a 26 años, todos ellos estudiantes del nivel de licenciatura de la carrera de Psicología, de los que se obtuvo información sobre su consumo de alcohol. La mayoría cursaba el primer semestre $(84 \%)$ y asistía a clases en el turno matutino (79\%). Los criterios de inclusión fueron los siguientes: ser mayores de 18 años, estudiantes universitarios y residentes en la Ciudad de México o zona metropolitana. A su vez, los criterios de exclusión fueron tener un diagnóstico de trastornos neurológicos o psiquiátricos, estar bajo tratamiento con hipnóticos o neurolépticos al momento de la evaluación, o presentar problemas físicos que impidieran la evaluación. Se identificó a 33 jóvenes que cumplían con el criterio de CEA (31.4\%), de los cuales se excluyeron 13 debido a que no cumplían con los criterios para continuar en el estudio, retiraron su consentimiento o se perdió la comunicación con ellos. Una vez conformado el grupo con patrón CEA (GCE, $n=20$ ), se conformó el grupo control con los jóvenes sin tal patrón, similares en sexo y edad al GCE $(\mathrm{GC}, \mathrm{n}=19)$. La muestra final se constituyó con 39 participantes: 15 hombres y 24 mujeres. En la Tabla 1 se presentan las características demográficas y de consumo de alcohol de ambos grupos. Como se esperaba, hubo diferencias significativas entre los grupos en términos de la frecuencia y cantidad de consumo de alcohol.

\section{Instrumentos}

Se utilizó una hoja de datos demográficos y de antecedentes patológicos personales y familiares mediante la cual se verificaron los criterios de inclusión y exclusión de los participantes. Además, 
Tabla 1. Características demográficas y de consumo de alcohol de la muestra estudiada.

\begin{tabular}{|c|c|c|c|}
\hline Característica demográfica & $\begin{array}{c}\text { GCE } \\
\text { n (\%) }\end{array}$ & $\begin{array}{c}\text { GC } \\
\text { n }(\%)\end{array}$ & $p$ \\
\hline Edad (D.E.) & $20.5(1.7)$ & $21.4(2.1)$ & $.164^{\mathrm{a}}$ \\
\hline \multicolumn{4}{|l|}{ Sexo: } \\
\hline Hombres & $9(45.0)$ & $6(31.0)$ & \multirow[t]{2}{*}{$.389^{b}$} \\
\hline Mujeres & $11(55.0)$ & $13(69.0)$ & \\
\hline \multicolumn{4}{|l|}{ Frecuencia de consumo de alcohol: } \\
\hline Nunca & $0(0)$ & $8(42)$ & \multirow[t]{3}{*}{$.001^{\mathrm{c}}$} \\
\hline Una vez al mes o menos & $11(55)$ & $11(57.9)$ & \\
\hline De dos a cuatro veces al mes & $9(45)$ & $0(0)$ & \\
\hline \multicolumn{4}{|l|}{ Número de copas ingeridas por ocasión: } \\
\hline 0 & $0(0)$ & $8(42.1)$ & \multirow[t]{6}{*}{$.001^{\mathrm{c}}$} \\
\hline $1-2$ & $0(0)$ & $9(47.4)$ & \\
\hline $3-4$ & $9(45)$ & $2(10.5)$ & \\
\hline $5-6$ & $4(20)$ & $0(0)$ & \\
\hline $7-9$ & $4(20)$ & $0(0)$ & \\
\hline 10 o más. & $3(15)$ & $0(0)$ & \\
\hline \multicolumn{4}{|c|}{ Frecuencia de consumo de seis copas o más en una ocasión: } \\
\hline Nunca. & $4(20)$ & $15(79)$ & \multirow[t]{3}{*}{$.002^{\mathrm{b}}$} \\
\hline Una vez al mes o menos. & $15(75)$ & $4(21)$ & \\
\hline Una vez a la semana o más. & $1(5)$ & $0(0)$ & \\
\hline
\end{tabular}

para descartar posibles alteraciones psiquiátricas en los participantes, se utilizó el Listado de Comprobación de Síntomas (SCL-90, por las siglas en inglés de Symptom Checklist-90). Es este un instrumento de autorreporte, con 90 reactivos que deben responderse en una escala Likert de 0 a 4 puntos. El instrumento proporciona un Índice de Severidad Global, que indica la probable existencia de un trastorno mental. El SCL-90 fue adaptado y validado en población mexicana por Cruz, Blas, González y Chávez (2005), quienes informaron de su alta consistencia interna y adecuada validez de constructo.

La división de los grupos de comparación se hizo con las preguntas 1, 2 y 3 del Test de Identificación de los Trastornos debidos al Consumo de Alcohol (AUDIT, por sus siglas en inglés), en la versión en español de Rubio, Bermejo, Caballero y Santo Domingo (1998). Dichas preguntas permiten identificar el consumo de riesgo de al- cohol según su frecuencia y cantidad (Saunders, Aasland, Babor, De la Fuente y Grant, 1993). El valor crítico se definió como haber tomado cinco copas de bebidas alcohólicas en el caso de los hombres, o cuatro para las mujeres en una misma ocasión, para lo cual se emplearon dos preguntas de la escala AUDIT para categorizar la muestra: “Cuántas copas tomas en un día que bebes?” y "¿Qué tan frecuentemente tomas seis o más copas en la misma ocasión?”. Quien cumplía el criterio se consideraba como un caso de CEA.

La evaluación de las funciones ejecutivas se llevó a cabo mediante la Batería Neuropsicológica de las Funciones Ejecutivas y los Lóbulos Frontales (BANFE), creada por Flores, Ostrosky y Lozano (2012). Su objetivo principal es la exploración de un amplio número de procesos cognitivos dependientes de diversas regiones de la corteza prefrontal. Consta de catorce subpruebas que evalúan funciones complejas que dependen de la corteza 
orbitofrontal y prefrontal medial (dominio orbitomedial), corteza prefrontal anterior (dominio prefrontal anterior) y corteza prefrontal dorsolateral (dominio dorsolateral). Las subpruebas incluidas en cada dominio son las siguientes:

\section{Dominio prefrontal orbitomedial}

Efecto Stroop. Evalúa la capacidad de inhibición y desempeño ante la interferencia.

Juego de cartas. Explora la capacidad de toma de decisiones respecto al análisis de riesgo-beneficio.

Laberintos (errores). Evalúa la capacidad de respetar reglas y seguir instrucciones.

\section{Dominio prefrontal anterior}

Clasificaciones semánticas. Estimula la creación de diferentes grupos semánticos explorando la capacidad de abstracción.

Refranes. Evalúa la comprensión, comparación y selección del sentido figurado.

Metamemoria. Brinda información sobre la predicción y los ajustes del propio desempeño en una prueba clásica de aprendizaje verbal.

\section{Dominio prefrontal dorsolateral}

Señalamiento autodirigido. Constituye una medida de memoria de trabajo visoespacial en la que el participante debe señalar una serie de figuras sin repetir.

Memoria de trabajo visoespacial. El participante debe retener y reproducir secuencialmente una serie de figuras.

Ordenamiento alfabético de palabras. Explora la memoria de trabajo verbal, ya que el sujeto debe ordenar mentalmente de forma alfabética una lista de palabras.

Clasificación de cartas. Medición clásica de flexibilidad cognitiva.

Laberintos (aciertos). Evalúan la capacidad de planeación visoespacial.

Torre de Hanoi. Explora la capacidad para planear de manera secuencial y bajo ciertas restricciones.

Suma y resta consecutivas. Evalúan la capacidad de secuenciación inversa.

Fluidez verbal. Indica la capacidad de producción verbal en un tiempo determinado.
Los procesos de aplicación y calificación de la BANFE se llevaron a cabo siguiendo las especificaciones del manual de la prueba, por lo que se utilizaron los valores normativos de población mexicana proporcionados por la misma.

\section{Procedimiento}

El presente estudio estuvo avalado por la Comisión de Ética de la Facultad de Estudios Superiores Iztacala de la Universidad Nacional Autónoma de México mediante el oficio CE/FESI/012020/1335. Se invitó a participar a estudiantes inscritos en una universidad pública del Estado de México de manera voluntaria. Los estudiantes interesados en formar parte del estudio fueron evaluados preliminarmente con el SCL-90, y asimismo se recolectaron datos demográficos y antecedentes patológicos personales y familiares para verificar los criterios de inclusión y exclusión. Si los participantes cumplían con estos criterios, se les daba una hoja de consentimiento informado para que fuera firmada por ellos. Posteriormente, se hacía la evaluación neuropsicológica, con una duración aproximada de 60 minutos. La información obtenida se capturó en una base de datos para su análisis estadístico, el cual se llevó a cabo con el paquete estadístico IBM SPSS, v. 20. Se contrastó la hipótesis de normalidad mediante el test Kolmogorov-Smirnov, utilizándose estadística paramétrica descriptiva para el análisis de los datos demográficos y consumo de alcohol. La comparación del desempeño neuropsicológico se efectuó con la prueba $t$ de Student, calculándose el tamaño del efecto con la prueba $g$ de Hedges.

\section{RESULTADOS}

La Tabla 2 muestra las puntuaciones normalizadas por dominio anatómico de ambos grupos según la BANFE. Aunque el GCE obtuvo puntuaciones menores que el GC en todas las áreas, se observaron diferencias significativas en el dominio dorsolateral $(t=-2.128, p=.040)$, que mostró un tamaño del efecto mediano $(g=.686)$, y en la puntuación total del instrumento $(t=-2.636, p=.012)$ con tamaño grande del efecto $(\mathrm{g}=.842)$. 
Tabla 2. Comparación de las puntuaciones normalizadas en ambos grupos por dominio anatómico de acuerdo con la BANFE.

\begin{tabular}{|l|c|c|c|c|}
\hline \multicolumn{1}{|c|}{ Dominio } & $\begin{array}{c}\text { GCE } \\
\text { M (D.E.) }\end{array}$ & $\begin{array}{c}\text { GC } \\
\text { Media } \\
\text { (D.E.) }\end{array}$ & $\boldsymbol{t}$ & $\boldsymbol{g}$ \\
\hline Orbitomedial & $91.6(32.9)$ & $98.8(12.1)$ & -.895 & 0.287 \\
\hline Prefrontal anterior & $103.2(10)$ & $105.7(7.8)$ & -.877 & 0.277 \\
\hline Dorsolateral & $75.5(18.5)$ & $88.2(18.5)$ & $-2.128^{*}$ & 0.686 \\
\hline \multicolumn{7}{|l|}{ Total } & $76.3(18)$ & $90.4(15.3)$ & $-2.636^{*}$ & 0.842 \\
\hline
\end{tabular}

Se analizaron las puntuaciones naturales obtenidas en las tareas que conforman cada área de la prueba para conocer la ejecución relacionada con procesos específicos del funcionamiento ejecutivo, observándose diferencias en la tarea de ordenamien- to alfabético de palabras, ensayo $3(t=-2.107$, $p=.042)$ y en la tarea de clasificación de tarjetas $(t=-2.198, p=.036)$. En ambos casos el GC tuvo una ejecución superior al GCE, siendo mediano el tamaño del efecto (Tabla 3).

Tabla 3. Comparación entre grupos de las puntuaciones naturales obtenidas en las tareas de la BANFE.

\begin{tabular}{|c|c|c|c|c|}
\hline Dominio & $\begin{array}{c}\text { GCE } \\
\text { M (D.E.) }\end{array}$ & $\begin{array}{c}\text { GC } \\
\text { M (D.E.) }\end{array}$ & $t$ & $g$ \\
\hline \multicolumn{5}{|l|}{ Orbitomedial } \\
\hline Stroop A - Aciertos & $79.7(4.8)$ & $77.5(14.4)$ & .652 & .207 \\
\hline Stroop A - Errores & $2.1(2.4)$ & $3.6(7.6)$ & 1.469 & .269 \\
\hline Stroop A - Tiempo & $7(17.2)$ & $1.3(0.2)$ & 1.483 & .462 \\
\hline Stroop B - Aciertos & $79.6(15.1)$ & $83.7(0.7)$ & -1.190 & .378 \\
\hline Stroop B - Errores & $1.9(5.6)$ & $0.1(0.3)$ & 1.469 & .447 \\
\hline Stroop B - Tiempo & $14.6(24)$ & $6.4(15.9)$ & 1.271 & .400 \\
\hline Juego de cartas - Porcentaje cartas riesgo & $29.4(11.7)$ & $34.8(11.1)$ & -1.480 & .473 \\
\hline Juego de cartas - Total & $32.9(17.8)$ & $37.4(22.5)$ & -.697 & .222 \\
\hline Clasificación de tarjetas - Errores & $0.5(1.2)$ & $0.7(0.8)$ & -.688 & .195 \\
\hline Laberintos - Errores & $1.2(1.6)$ & $0.5(1.2)$ & 1.320 & .493 \\
\hline \multicolumn{5}{|l|}{ Prefrontal anterior } \\
\hline Clasificación semántica & $21.7(6.4)$ & $21.9(5.4)$ & -.129 & .033 \\
\hline Refranes - Aciertos & $4.2(0.7)$ & $5.8(8.3)$ & -.855 & .275 \\
\hline Refranes - Tiempo & $18.9(23.2)$ & $15.7(23)$ & .427 & .138 \\
\hline Metamemoria - Error negativo & $2.7(1.9)$ & $2.3(1.4)$ & .672 & .230 \\
\hline Metamemoria - Error positivo & $1.2(1.3)$ & $1.2(1.2)$ & -.154 & 0 \\
\hline \multicolumn{5}{|l|}{ Dorsolateral } \\
\hline Señalamiento autodirigido - Aciertos & $19.9(4.1)$ & $18.4(4)$ & 1.126 & .370 \\
\hline Señalamiento autodirigido - Perseveración & $2.7(2)$ & $2.6(1.8)$ & .025 & .052 \\
\hline Señalamiento autodirigido - Tiempo & $10.6(18.3)$ & $7(13.3)$ & .706 & .224 \\
\hline Memoria trabajo visoespacial - Nivel & $2.8(0.9)$ & $2.4(0.8)$ & 1.508 & .469 \\
\hline Memoria trabajo visoespacial - Perseveración & $0.1(0.3)$ & $0(0.2)$ & 1.000 & .390 \\
\hline Memoria trabajo visoespacial - Errores orden & $1.9(1.9)$ & $3(2.7)$ & -1.355 & .451 \\
\hline Ordenamiento - Ensayo 1 & $1.6(0.8)$ & $1.6(1)$ & .059 & .000 \\
\hline Ordenamiento - Ensayo 2 & $2.9(1.2)$ & $2.8(1.3)$ & .135 & .000 \\
\hline
\end{tabular}




\begin{tabular}{|l|r|r|r|c|}
\hline Ordenamiento - Ensayo 3 & $2.3(1.4)$ & $3.4(1.7)$ & $-2.107^{*}$ & .708 \\
\hline Clasificación tarjetas - Aciertos & $42.9(12.3)$ & $49.7(6.2)$ & $-2.198^{*}$ & .692 \\
\hline Clasificación tarjetas - Perseveraciones & $3.1(4.4)$ & $1.4(1.6)$ & 1.631 & .508 \\
\hline Clasificación tarjetas - Perseveraciones criterio & $0(0.2)$ & $0(0)$ & .974 & \multicolumn{1}{c|}{ NA } \\
\hline Clasificación tarjetas - Tiempo & $24.2(87.5)$ & $13.7(40.2)$ & .481 & .152 \\
\hline Clasificación semántica - Categorías & $8.8(2.3)$ & $9(2.5)$ & -.254 & .083 \\
\hline Clasificación semántica - Promedio & $7.2(7.4)$ & $5.1(1.3)$ & 1.220 & .390 \\
\hline Clasificación semántica - Total & $21.7(6.4)$ & $21.9(5.4)$ & -.129 & .033 \\
\hline Laberintos - Planeación & $2.2(2.7)$ & $2(2.2)$ & .246 & .000 \\
\hline Laberintos - Tiempo & $94.8(48.1)$ & $86.8(49)$ & .512 & .164 \\
\hline Torre 3 - Movimientos & $12.4(7.1)$ & $12.2(6.3)$ & .110 & .029 \\
\hline Torre 3 - Tiempo & $19.6(18.8)$ & $23.8(20.2)$ & -.666 & .215 \\
\hline Torre 4 - Movimientos & $30.4(22.7)$ & $31.7(12.2)$ & -.227 & .070 \\
\hline Torre 4 - Tiempo & $22.1(42.1)$ & $11.6(18.9)$ & .996 & .318 \\
\hline Suma - Aciertos & $16.9(6.6)$ & $18.6(4.2)$ & -.973 & .305 \\
\hline Suma - Tiempo & $31.5(19)$ & $31.1(19)$ & .074 & .021 \\
\hline Resta a partir de 100 - Aciertos & $9.9(2.7)$ & $11.2(2.8)$ & -1.443 & .472 \\
\hline Resta a partir de 100 - Tiempo & $11.2(14.6)$ & $19(22.7)$ & -1.280 & .411 \\
\hline Resta a partir de 40 - Aciertos & $10.2(3)$ & $10.5(3)$ & -.339 & .100 \\
\hline Resta a partir de 40 - Tiempo & $25(18.3)$ & $33.8(17)$ & -1.547 & .497 \\
\hline Fluidez verbal - Aciertos & $20.7(7.9)$ & $19.6(6.9)$ & .444 & .148 \\
\hline Fluidez verbal - Perseveraciones & $0.6(0.8)$ & $0.3(0.7)$ & 1.128 & .398 \\
\hline GCE = Grupo de consumo excesivo de alcohol; GC = Grupo control; M = Media; D.E. = Desviación estándar. \\
\hline * $<.05$. & & & & \\
\hline
\end{tabular}

\section{DISCUSIÓN}

El objetivo del presente estudio fue evaluar el funcionamiento ejecutivo de jóvenes con patrón CEA y comparar su desempeño con un grupo de jóvenes sin este. Los resultados muestran un efecto negativo del CEA sobre el perfil de funcionamiento ejecutivo. La disminución en el puntaje total de la BANFE, así como las bajas puntuaciones en el dominio dorsolateral del instrumento, son consistentes con los estudios que plantean que el CEA tiene un efecto negativo en las funciones ejecutivas en jóvenes, probablemente asociadas a un retraso madurativo de los lóbulos frontales (Hartley et al., 2004; Houston et al., 2014; Lannoy et al., 2019; Lannoy et al., 2018; Lees et al., 2019; Mota et al., 2013; Parada et al., 2012; Salas et al., 2016; Scaife y Duka, 2009; Townshed y Duka, 2005).

Además, dichos resultados son compatibles con los estudios de neuroimagen que han encontrado diferencias en los patrones de maduración prefrontal en jóvenes con CEA. Hay evidencia robusta de la reducción del volumen de materia blanca en áreas prefrontales en esta población, lo que se correlaciona con las fallas en el funcionamiento ejecutivo y en la conducta (Clark, Thatcher y Tapert, 2008; Cservenka y Brumback, 2017; Hermens et al., 2013; Jacobus y Tapert, 2013; Lees, Meredith, Kirkland, Bryant y Squeglia, 2020; Petit, Maurage, Kornreich, Verbanck y Campanella, 2014; Siqueira, Smith y Committee on Substance Abuse, 2015; Squeglia et al., 2014). Respecto a las diferencias halladas en el dominio prefrontal dorsolateral, también hay consistencia con otros estudios que han explorado el funcionamiento de esta área a través de la evaluación neuropsicológica (Mota et al., 2013; Parada et al., 2012; Scaife y Duka, 2009), así como con resonancia magnética funcional (Ames et al., 2014; Cservenka y Brumback, 2017; Dager et al., 2014).

Los resultados del presente estudio muestran en consecuencia que el funcionamiento ejecutivo 
general podría estar comprometido en estos jóvenes. Si se analizan las puntuaciones de las tareas que conforman la prueba, se puede observar que el desempeño de los participantes con CEA fue consistentemente más pobre que el del grupo control, aunque no hubo diferencias significativas en todas las tareas. Sin embargo, la ponderación del índice general que ofrece el instrumento muestra una clara diferencia entre los grupos. Aunado a ello, la diferencia observada respecto al funcionamiento prefrontal dorsolateral proporcionado por la batería señala que las tareas relacionadas con estas regiones (memoria de trabajo, flexibilidad cognitiva, planeación, etc.) no se ejecutaron al nivel esperado según su grupo de comparación.

El análisis por tarea ofrece información relevante en cuanto que hubo diferencias en el ensayo 3 de la prueba de ordenamiento alfabético de palabras y el número de aciertos de la tarea de clasificación de tarjetas; estas pruebas se asocian con memoria de trabajo verbal y flexibilidad cognitiva. El bajo desempeño de los jóvenes CEA en ambas es un hallazgo consistente con lo reportado en otras investigaciones (García, Expósito, Sanhueza y Angulo, 2008; García, Expósito, Sanhueza y Gil, 2009; Parada et al., 2012; Sanhueza, García y Expósito, 2011). Sin embargo, es importante mencionar que otros estudios han encontrado afectaciones en la memoria de trabajo visoespacial, principalmente (Scaife y Duka, 2009; Townshed y Duka, 2005). En una revisión relativamente reciente de Carbia et al. (2018) se señala que el desempeño en tareas de memoria de trabajo verbal es similar entre jóvenes CEA y controles. Al respecto, tales autores plantean que las tareas clásicas de memoria de trabajo verbal (p. e. span de dígitos en progresión y regresión) podrían no ser sensibles en esta población ya que su demanda cognitiva es baja. Es probable que la prueba utilizada en el presente estudio (ordenamiento alfabético de palabras) implicara una mayor exigencia cognitiva. En ella, el sujeto debe ordenar alfabéticamente una lista de palabras. Se le presentan tres listas progresivamente demandantes: la primera consta de cinco palabras que empiezan con vocales; la segunda contiene seis palabras que empiezan con consonantes, y la tercera incluye siete palabras que empiezan con consonante o vocal. Se asume que esta tarea es más demandante que otras porque hay varios procesos involucrados para realizarla de manera exitosa, como el automonitoreo (Flores et al., 2012). Es interesante que en el presente estudio se hallaran diferencias significativas solamente en la tercera lista (ensayo 3), la cual es más compleja y, por lo tanto, demanda mayores recursos cognitivos.

Respecto a la flexibilidad cognitiva, la evidencia también es controversial. En la revisión de Lopez et al. (2014) se afirma que dicha flexibilidad no parece estar afectada en jóvenes con patrón CEA, aunque algunos estudios han señalado la ocurrencia de alteraciones en función del sexo, siendo las mujeres el grupo que muestra un desempeño más pobre en tareas que evalúan este proceso (Hartley et al., 2004; Scaife y Duka, 2009). En la revisión de Carbia et al. (2018) se señalan las recientes evidencias sobre las alteraciones en la flexibilidad cognitiva, y se afirma que las inconsistencias entre los diversos estudios pueden deberse a las diferentes tareas utilizadas para evaluar este dominio. Los presentes resultados aportan evidencia sobre una menor capacidad de flexibilidad cognitiva observada en jóvenes con patrón CEA. No fue posible realizar comparaciones fiables en función del sexo que permitieran aportar más información debido al tamaño de la muestra.

Este estudio exploró la relación entre el patrón CEA y las funciones ejecutivas en una muestra de universitarios mexicanos con un instrumento específico del funcionamiento ejecutivo, lo que permitió evaluar a profundidad los dominios cognitivos asociados con los lóbulos frontales. Tal instrumento, además de específico, cuenta con normas de la población mexicana, por lo que resultó sensible para evaluar la población en cuestión. A juicio de los presentes autores, no hay estudios que hayan evaluado la relación entre el patrón CEA y las funciones ejecutivas en universitarios mexicanos. De cualquier manera, se encontraron resultados consistentes con la literatura internacional que describe los efectos negativos del CEA sobre el funcionamiento ejecutivo. En este trabajo se halló que funciones relacionadas con la corteza prefrontal dorsolateral, como la memoria de trabajo verbal y la flexibilidad cognitiva, parecen estar especialmente afectadas en esta población. Las 
tareas de memoria de trabajo verbal que implican una mayor demanda cognitiva podrían ser más sensibles para evaluar los efectos del CEA en esta población, por lo que se recomienda su utilización en futuras investigaciones. Es así como aquí se aporta evidencia sobre los efectos del consumo excesivo de alcohol en el funcionamiento cognitivo de estudiantes universitarios, lo que debe tomarse en consideración para la realización de programas de prevención, psicoeducación o intervención en poblaciones vulnerables como las de los adolescentes y adultos jóvenes.

Aun así, el presente estudio padece algunas limitaciones importantes, como el tamaño de la muestra, razón por la cual los resultados deben ser tomados con precaución; por otro lado, no se tomó en cuenta el consumo de otras sustancias, como marihuana o cocaína, que tienen un importante efecto en el funcionamiento ejecutivo (Bava y Tapert, 2010; Siqueira et al., 2015). Por último, el alcance del estudio es correlacional, es decir, no es posible establecer una relación causal entre el CEA y las alteraciones en el funcionamiento ejecutivo. Es posible que la falta de maduración cerebral -que podría verse reflejada indirectamente en el funcionamiento ejecutivo- ocasione que los jóvenes consuman alcohol en exceso, en lugar de que tal consumo tenga efectos sobre el desarrollo cerebral y se refleje en ciertas alteraciones funcionales cognitivas (Hermens et al., 2013).
Sin embargo, algunos estudios con animales han evidenciado que el consumo de alcohol tiene efectos en el desarrollo del sistema nervioso durante la adolescencia (Crews, Braun, Hoplight, Switzer y Knapp, 2000; Silvers, Tokunaga, Mittleman y Matthews, 2003); además, los cambios anatomofuncionales relacionados con el consumo de riesgo CEA parecen ser similares a los observados en el alcoholismo crónico (Bates, Bowden y Barry, 2002; Clark et al., 2008). Ambas líneas de investigación apuntan a que el consumo excesivo de alcohol tiene consecuencias directas en el desarrollo del sistema nervioso. Para futuros estudios se sugiere evaluar muestras más grandes, de manera longitudinal y tomando en cuenta el consumo de otras sustancias, lo que permitirá obtener información de mayor alcance.

En conclusión, el CEA tiene un efecto negativo generalizado sobre el funcionamiento ejecutivo en jóvenes universitarios; en particular, las funciones asociadas con la corteza dorsolateral parecen especialmente sensibles. Estos datos resultan congruentes con la hipótesis de la vulnerabilidad del lóbulo frontal ante el consumo excesivo de alcohol. Los presentes hallazgos tienen implicaciones clínicas importantes debido a que la disminución en la función cognitiva de los jóvenes, aunada a la baja percepción de riesgo por el consumo, podría aumentar el riesgo para desarrollar un trastorno por uso de sustancias.

Citación: Mondragón-Maya, A., Flores-Medina, Y., López-Arreaga., G., López-Ramírez, S. y Paz-Rodríguez, F. (2021). Funciones ejecutivas en estudiantes universitarios con patrón de consumo excesivo de alcohol. Psicología y Salud, 31(2), 295-306. https://doi.org/10.25009/pys.v31i2.2698.

\section{REFERENCIAS}

Ames, S.L., Wong, S.W., Bechara, A., Cappelli, C., Dust, M., Grenard, J.L. y Stacy, A.W. (2014). Neural correlates of a Go/NoGo task with alcohol stimuli in light and heavy young drinkers. Behavioral and Brain Research, 274, 382-389. Doi: 10.1016/j. bbr.2014.08.039.

Bates, M., Bowden, S.C. y Barry, D. (2002). Neurocognitive impairment associated with alcohol use disorders: Implications for treatment. Experimental and Clinical Psychopharmacology, 10(3), 193-212. Doi: 10.1037//1064-1297.10.3.193.

Bava, S. y Tapert, S.F. (2010). Adolescent brain development and the risk for alcohol and other drug problems. Neuropsychological Reviews, 20, 398-413. Doi: 10.1007/s11065-010-9146-6.

Boelema, S., Harakeh, Z., van Zandvoort, M.J.E., Reijneveld, S A., Verhulst, F.C., Ormel, J. y Vollebergh, W.A.M. (2015). Adolescent heavy drinking does not affect maturation of basic executive functioning: Longitudinal findings from the TRAILS study. PloS ONE, 10(10) (Artículo e0139186). Doi: 10.1371/journal. pone.0139186.

Carbia, C., López C., E., Corral, M. y Cadaveira, F. (2018). A systematic review of neuropsychological studies involving young binge drinkers. Neuroscience \& Biobehavioral Reviews, 90, 332-349. Doi: 10.1016/j.neubiorev.2018.04.013. 
Chung, T., Creswell, K.G., Bachrach, R., Clark, D.B. y Martin, C.S. (2018). Adolescent binge drinking: Developmental context and opportunities for prevention. Alcohol Research: Current Reviews, 39(1), 5-15. Recuperado de psycnet.apa.org/record/2019-05003-001.

Clark, D.B., Thatcher, D.L. y Tapert, S.F. (2008). Alcohol, psychological dysregulation, and adolescent brain development. Alcoholism: Clinical and Experimental Research, 32(3), 375-385. Doi: 10.111/j.1530-0277.2007.00601.x.

Crews, F.T., Braun, C.J., Hoplight, B., Switzer, R.C. y Knapp, D.J. (2000). Binge ethanol consumption causes differential brain damage in young adolescent rats compared with adult rats. Alcoholism: Clinical and Experimental Research, 24(11), 17121723. Recuperado de pubmed.ncbi.nlm.nih.gov/11104119/.

Cruz C., S., López, L., Blas, C., González, L. y Chávez R., A. (2005). Datos sobre validez y confiabilidad de la Symptom Check List 90 (SCL 90) en una muestra de sujetos mexicanos. Salud Mental, 28(1), 72-81. Recuperado de http://www.redalyc.org/ articulo.oa? id=58212808.

Cservenka, A. y Brumback, T. (2017). The burden of binge and heavy drinking on the brain: Effects on adolescent and young adult neural structure and function. Frontiers in Psychology, 8, Article 1111. Doi: 10.3389/fpsyg.2017.01111.

Dager, A.D., Jamadar, S., Stevens, M.C., Rosen, R., Jiantonio-Kelly, R.E., Sisante, J.F., Raskin, S.A., Tennen, H., Austad, C.S., Wood, R.M., Fallahi, C.M. y Pearlson, G.D. (2014). FMRI response during figural memory task performance in college drinkers. Psychopharmacology, 231, 167-179. Doi: 10.1007/s00213-013-3219-1.

Diamond, A. (2013). Executive functions. Annual Reviews in Psychology, 64, 135-168. Doi: 10.1146/annurev-psych-113011143750 .

Flores, J.C., Ostrosky S., F. y Lozano, A. (2012). Batería de funciones frontales y ejecutivas (BANFE). México: El Manual Moderno.

García M., L.M., Expósito, J., Sanhueza, C. y Angulo, M. (2008). Actividad prefrontal y alcoholismo de fin de semana en jóvenes. Adicciones, 20, 271-279. Recuperado de http://www.adicciones.es/index.php/adicciones/article/view/269/256.

García M., L.M., Expósito, J., Sanhueza, C. y Gil, S. (2009). Rendimiento cognitivo y alcoholismo de fin de semana en adolescentes. Neuropsicología, Neuropsiquiatría y Neurociencias, 9, 75-91.

Haller, M., Handley, E., Chassin, L. y Bountress, K. (2010). Developmental cascades: academic achievement to adult substance use disorder. Developmental Psychopathology, 22(4), 899-916. Doi: 10.1017/S0954579410000532.

Hartley, D.E., Elsabagh, S. y File, S.E. (2004). Binge drinking and sex: Effects on mood and cognitive function in healthy young volunteers. Pharmacology, Biochemistry and Behavior, 78, 611-619. Doi: 10.1016/j.pbb.2004.04.027.

Hendricks, H., van de Rest, O., Snippe, A., Kieboom, J. y Hogenelst, K. (2020). Alcohol consumption, drinking patterns, and cognitive performance in young adults: A cross-sectional and longitudinal analysis. Nutrients, 12, Article 200. Doi: 10.3390/ nu12010200.

Hermens, D.F., Lagopoulos, J., Tobias-Webb, J., De Regt, T., Dore, G., Juckes, L., Latt, N. y Hickie, I.B. (2013). Pathways to alcohol-induced brain impairment in young people: A review. Cortex, 49, 3-17. Doi: 10.1016/j.cortex.2012.05.021.

Houston, R.J., Derrick, J.L., Leonard, K.E., Testa, M., Quigley, B.M. y Kubiak, A. (2014). Effects of heavy drinking on executive cognitive functioning in a community sample. Addictive Behaviors, 39, 345-349. Doi: 10.1016/j.addbeh.2013.09.032.

Jacobus J. y Tapert, S.F. (2013). Neurotoxic effects of alcohol in adolescence. Annual Reviews in Clinical Psychology, 9, 703-721. Doi: 10.1146/annurev-clinpsy-050212-185610.

Kulis S., S., Marsiglia F., F., Nuño G., B.L., Lozano M., D. y Medina-Mora, M.E. (2018). Traditional gender roles and substance-use behaviors, attitudes, exposure, and resistance among early adolescents in large cities of Mexico. Journal of Substance Use, 23(5), 471-480. Doi: 10.1080/14659891.2017.1405088.

Kuntsche, E., Kuntsche, S., Thrul, J. y Gmel, G. (2017). Binge drinking: Health impact, prevalence, correlates and interventions. Psychology and Health, 1-42. Doi: 10.1080/08870446.2017.1325889.

Lannoy, S., Billieux, J., Dormal, V. y Maurage, P. (2019). Behavioral and cerebral impairments associated with binge drinking in youth: A critical review. Psychologica Belgica, 59(1), 116-155. Doi: 10.5334/pb.476.

Lannoy, S., Maurage, P., D’Hont, F., Billieux, J. y Dormal, V. (2018). Executive impairments in binge drinking: Evidence for a specific performance-monitoring difficulty during alcohol-related processing. European Addiction Research, $24,118-127$. Doi: 10.1159/000490492.

Lebel, C. y Beaulieu, C. (2011). Longitudinal development of human brain wiring continues from childhood into adulthood. The Journal of Neuroscience, 31, 10937-10947. Doi: 10.1111/j.1530-0277.2002.tb02597.x.

Lees, B., Meredith, L.R., Kirkland, A.E., Bryant, B.E. y Squeglia, L.M. (2020). Effect of alcohol use on the adolescent brain and behavior. Pharmacology Biochemistry and Behavior, 192, Article 172906. Doi: 10.1016/j.pbb.2020.172906.

Lees, B., Mewton, L., Stapinski, L.A., Squeglia, L.M., Rae, C.D. y Teesson, M. (2019). Neurobiological and cognitive profile of young binge drinkers: A systematic review and meta-analysis. Neuropsychology Review, 29, 357-385. Doi: 10.1007/ s11065-019-09411-w. 
López C., E., Mota, N., Crego, A., Velasquez, T., Corral, M., Rodríguez, S. y Cadaveira, F. (2014). Anomalías neurocognitivas asociadas al consumo excesivo de alcohol (binge drinking) en jóvenes y adolescentes: una revisión. Adicciones, 26(4), 334-359. Recuperado de http://www.adicciones.es/index.php/adicciones/article/view/39.

Mahedy, L., Suddell, S., Skirrow, C., Fernandes, G.S., Field, M., Heron, J., Hickman, M., Wootton, R. y Munafò, M. R. (2020). Alcohol use and cognitive functions in young adults: Improving casual inference. Addiction. Doi: 10.1111/add.15100.

Monteiro, M.G. (2007). Alcohol and public health in the Americas. A case for action. Washington, D.C.: World Health Organization. Recuperado de www.who.int/substance_abuse/publications/alcohol_public_health_americas.pdf.

Mota, N., Parada, M., Crego, A., Doallo, S., Caamaño I., F., Rodríguez, S., Cadaveira, F. y Corral, M. (2013). Binge drinking trajectory and neuropsychological functioning among university students: A longitudinal study. Drug and Alcohol Dependence, 133, 108-114. Doi: 10.1016/j.drugalcdep.2013.05.024.

Parada, M., Corral, M., Mota, N., Crego, A., Rodriguez, S. y Cadaveira, F. (2012). Executive function and alcohol binge drinking in university students. Addictive Behaviors, 37, 167-172. Doi: 10.1016/j.addbeh.2011.09.015.

Petit, G., Maurage, P., Kornreich, C., Verbanck, P. y Campanella, S. (2014). Binge drinking in adolescents: A review of neurophysiological and neuroimaging research. Alcohol and Alcoholism, 49(2), 198-206. Doi: 10.1093/alcalc/agt172.

Reséndiz, E., Bustos, M.N., Mújica, R., Soto, I.S., Cañas, V., Fleiz, C., Gutiérrez, M.L., Amador, N., Medina-Mora, M.E. y Villatoro, J.A. (2018). National trends in alcohol consumption in Mexico: Results of the National Survey on Drug, Alcohol and Tobacco Consumption 2016-2017. Salud Mental, 41(1), 7-15. Doi: 10.17711/SM.0185-3325.2018.003.

Rubio, G., Bermejo, J., Caballero, M.C. y Santo Domingo, J. (1998). Validación de la prueba para la identificación de trastornos por uso de alcohol (AUDIT) en atención primaria. Revista Clínica Española, 198, 11-14.

Salas G., D., Fernandez G., M., Pozueta, A., Diaz C., I., Lamarain, M., Perez, C. y Sanchez J., P. (2016). Binge drinking in young university students is associated with alterations in executive functions related to their starting age. PLOS ONE, 11(11), Article e0166834. Doi: 10.1371/journal.pone.0166834.

Sanhueza, C., García M., L.M. y Expósito, J. (2011). Weekend alcoholism in youth and neurocognitive aging. Psicothema, 23, 209-214.

Saunders, J.B., Aasland, O.G., Babor, T.F., De la Fuente, J.R. y Grant, M. (1993). Development of the Alcohol Use Disorders Identification Test (AUDIT): WHO Collaborative Project on Early Detection of Persons with Harmful Alcohol Consumption-II. Addiction, 88(6), 791-804.

Scaife, J.C. y Duka, T. (2009). Behavioral measures of frontal lobe function in a population of young social drinkers with binge drinking pattern. Pharmacology, Biochemistry and Behavior, 93, 354-362. Doi: 10.1016/j.pbb.2009.05.015.

Secretaría de Salud, Instituto Nacional de Salud Pública e Instituto Nacional de Estadística y Geografía (2018). Encuesta Nacional de Salud y Nutrición (ENSANUT 2018). Ciudad de México: Autores. Recuperado de ensanut.insp.mx/encuestas/ensanut2018/doctos/informes/ensanut_2018_presentacion_resultados.pdf.

Silvers, J.M., Tokunaga, S., Mittleman, G. y Matthews, D.B. (2003). Chronic intermittent injections of high-dose ethanol during adolescence produce metabolic, hypnotic, and cognitive tolerance in rats. Alcoholism: Clinical and Experimental Research, 27(10), 1606-1612. Doi: 10.1097/01.ALC.0000090141.66526.22.

Siqueira, L., Smith, V. y Committee on Substance Abuse (2015). Binge drinking. Pediatrics, 136(3), e718-e726. Doi: 10.1542/ peds.2015-2337.

Squeglia, L.M., Jacobus, J. y Tapert, S.F. (2014). The effect of alcohol use on human adolescent brain structures and systems. Handbook of Clinical Neurology, 125, 501-510. Doi: 10.1016/B978-0-444-62619-6.00028-8.

Squeglia, L.M., Schweinsburg, A.D., Pulido, C. y Tapert, S.F. (2011). Adolescent binge drinking linked to abnormal spatial working memory brain activation: Differential gender effects. Alcoholism: Clinical and Experimental Research, 35(10), 1831-1841. Doi: 10.1111/j.1530-0277.2011.01527.x.

Squeglia, L.M., Sorg, S.F., Schweinsburg, A.D., Wetherill, R.R., Pulido, C. y Tapert, S.F. (2012). Binge drinking differentially affects adolescent male and female brain morphometry. Psychopharmacology, 220(3), 529-539. Doi: 10.1007/s00213-011$2500-4$.

Stuss, D.T. y Alexander, M.P. (2000). Executive functions and the frontal lobes: A conceptual view. Psychological Research, 63(3-4), 289-298. Doi: 10.1007/s004269900007.

Townshed, J.M. y Duka, T. (2005). Binge drinking, cognitive performance and mood in a population of young social drinkers. Alcoholism: Clinical and Experimental Research, 29(3), 317-325.

Wechsler, H., Davenport, A., Dowdell, G. y Rimm, E.B.A. (1995). Gender specific measure of binge drinking among college students. American Journal of Public Health, 85(7), 982.

Wechsler, H., Lee, J.E., Kuo, M., Seibring, M., Nelson, T.F. y Lee, H. (2002). Trends in college binge drinking during a period of increased prevention efforts. Findings from 4 Harvard School of Public College Alcohol Study surveys: 1993-2001. Journal of the American College Health, 50, 203-217. Doi: 10.1080/07448480209595713. 